Classification

Physics Abstracts

75.25

\title{
Squaring-up of modulated magnetic structures in HoAg and TmAg
}

\author{
P. Morin, D. Schmitt, \\ Laboratoire Louis Néel, C.N.R.S., 166X, 38042 Grenoble Cedex, France \\ and C. Vettier \\ Institut Laue-Langevin, 156X, 38042 Grenoble Cedex, France
}

(Reçu le 20 avril 1984, accepté le 27 septembre 1984)

\begin{abstract}
Résumé. - La dépendance thermique des structures magnétiques modulées en amplitude de HoAg et TmAg a été étudiée par diffraction neutronique sur des monocristaux. Leur évolution vers un régime antiphase a été observée à basse température, comme attendu, mais les conditions expérimentales ont empêché ce régime d'être complètement atteint. Aucun blocage du vecteur de propagation sur une valeur commensurable n'a été mis en évidence dans les limites de la précision expérimentale, aucune modulation du réseau via le couplage magnétoélastique n’a été détectée.

Abstract. - The temperature variation of the amplitude modulated magnetic structures of HoAg and TmAg has been investigated by neutron diffraction on single crystals. A beginning of squaring-up has been observed, but due to experimental conditions the expected antiphase regime could not be fully reached. No locking of the incommensurate propagation vector into commensurate values has been evidenced within the experimental accuracy and no lattice modulation through the magnetoelastic coupling has been detected.
\end{abstract}

\section{Introduction.}

Modulated magnetic structures can be found in many systems such as rare-earth metals [1] or rare-earth intermetallic compounds [2]. In transition element compounds such magnetic structures, corresponding to a spin density wave as in metallic chromium [3], or in magnetic insulators as $\mathrm{NiBr}_{2}[4]$ and $\mathrm{RbFeCl}_{3}[5]$, can be considered as exceptions. This is usually explained by the occurrence of maximum of $J(q)$ (the Fourier transform of the exchange interactions) for an incommensurate wave vector $\mathbf{q}_{\mathbf{m}}$ [6]. These magnetic structures may be phase modulated, e.g. spiral structures, or amplitude modulated. The latter case occurs when a strong magnetic anisotropy confines the magnetic moment along a given direction. When the temperature decreases below the Néel temperature $T_{\mathrm{N}}$, the modulated structures can change according to three distinct ways :

i) A first-order phase transition towards a commensurate magnetic structure can occur at a temperature $T_{\mathrm{t}}<T_{\mathrm{N}}$, with $T_{\mathrm{t}} / T_{\mathrm{N}}$ usually in the range $0.5-1$; this is the case of $\mathrm{TbZn}_{2}$ [7], $\mathrm{TbAu}_{2}$ [8], $\mathrm{ErCu}$ and $\mathrm{TmCu}$ [9] for example. This can be considered as a border-line case of the locking phenomenon, where $\mathbf{q}_{\mathbf{m}}$ locks into a commensurate value (rational fraction of a basis vector) over a finite temperature range, as for example in thulium metal [10].

ii) When the ground state is non-magnetic, e.g. non-Kramers ions or Kondo systems, the modulated structure can remain stable down to $0 \mathrm{~K}$ as it has been seen in $\mathrm{HoNi}_{0.5} \mathrm{Cu}_{0.5}$ [11], in $\mathrm{CeAl}_{2}$ [12] or in $\mathrm{TmS}$ [13].

iii) In the case of magnetic ground state (Kramers ions), entropy effects prevent the magnetic structure to remain modulated at low temperature : it must move towards an arrangement with constant magnetic moments, i.e. an antiphase structure [14]; this squaring-up was well demonstrated in Tm metal [15].

In a neutron diffraction experiment, modulated magnetic structures are evidenced by the occurrence of magnetic satellites at incommensurate wave vectors $\mathbf{q}= \pm \mathbf{q}_{\mathbf{m}}+\mathbf{G}$, $\mathbf{G}$ being a reciprocal lattice vector. Their intensity is associated with the amplitude of the order parameter $\mathbf{m}_{\mathbf{q}_{\mathbf{m}}}$, the first-order Fourier component of the magnetic moment $\mathbf{r n}(\mathbf{R})$. When the temperature is lowered, the higher order harmonics $\mathbf{m}_{n q_{m}}$ in the Fourier expansion of $\mathbf{m}(\mathbf{R})$ become more important in the free energy, corresponding to the squaringup of the magnetic structure, and leading to higher order magnetic satellites at wave vectors $q= \pm n q_{m}+G$. 
In the antiphase regime the intensity of these higher order satellites saturates, their relative values corresponding to the Fourier expansion of a square wave.

An interesting feature may be associated with amplitude modulated structures when a magnetoelastic coupling is present. Indeed together with the modulated magnetic moment, the $4 \mathrm{f}$ quadrupoles $Q$ also are modulated, but with a half period since the quadrupoles are unchanged when the magnetic moments change their sign. Therefore, the magnetoelastic coupling must give rise to i) a macroscopic average distortion due to the non-zero average value of $Q$, and ii) a lattice modulation with a wave vector twice as large as the magnetic incommensurate wave vector $\mathbf{q}_{\mathrm{m}}$. This magnetoelastic modulation could be evidenced in a neutron diffraction experiment by the occurrence of second-order satellites of nuclear origin at $\mathbf{q}= \pm 2 \mathbf{q}_{\mathrm{m}}+\mathbf{G}$. To our knowledge this has not yet been observed experimentally in rare-earth systems. In the case of a modulated magnetic structure squaring-up at low temperature, there exists an additional feature : indeed in the antiphase regime the quadrupoles are all constant in amplitude and sign, removing the incommensurability of the quadrupoles arrangement. Therefore, the lattice modulation and the secondorder satellites must vanish. The temperature dependence of the intensity of these satellites then must present a maximum between the antiphase regime and $T_{\mathrm{N}^{*}}$

The equiatomic rare-earth silver series, which crystallizes within the cubic CsCl-type structure, presents several cases of amplitude modulated magnetic structures, namely $\mathrm{HoAg}\left(T_{\mathrm{N}}=33 \mathrm{~K}\right)$ [16], $\operatorname{ErAg}$ [17] and $\operatorname{TmAg}\left(T_{\mathrm{N}}=9.5 \mathrm{~K}\right)$ [18]. While ErAg belongs to the first of the three groups quoted above $\left(T_{\mathrm{t}}=9.5 \mathrm{~K}, T_{\mathrm{N}}=18 \mathrm{~K}\right), \mathrm{HoAg}$ and $\mathrm{TmAg}$ were suspected to belong to the third group. Indeed no transition was detected down to $4.5 \mathrm{~K}$ for $\mathrm{HoAg}$ $\left(T / T_{N}=0.14\right)$ and $1.5 \mathrm{~K}$ for $\operatorname{TmAg}\left(T / T_{\mathrm{N}}=0.16\right)$. On the other hand, crystal field determinations proved the magnetic character of the ground state which is the triplet $\Gamma_{5}^{(1)}$ for both compounds $[18,19]$ : so they cannot belong to the second group.

The amplitude modulated magnetic structures of $\mathrm{HoAg}$ and $\mathrm{TmAg}$ have been previously determined by neutron diffraction on polycrystalline material $[16$, $18]$. We present here additional results of a neutron diffraction experiment performed on single crystals (sections 2 and 3) in order to study the temperature variation of the incommensurate wave vector, and of the higher order satellites associated with the squaring-up of the magnetic structure. A discussion is made in section 4.

\section{HoAg.}

2.1 EXPERIMENTAL. - The neutron diffraction experiments were carried out at the Institut Laue-Langevin in Grenoble, on the 4-circle spectrometer D10 used in a three-axis mode. PG (002) monochromator (inci- dent wavelength $\lambda=2.36 \AA$ ) and analyser were used with a PG filter in order to reduce high-order contamination. The HoAg single crystal was mounted in a continuous flow He-cryostat. The elastic peaks were investigated by $q$-scans in the temperature range 4.7-35 $\mathrm{K}$ with a resolution better than 0.01 in reduced units. The lattice parameter was found to be $a=$ $3.571 \AA$ at $11 \mathrm{~K}$.

2.2 Results. - The magnetic structure previously obtained [16] has been confirmed : in the entire ordered range $\mathrm{HoAg}$ has an amplitude modulated magnetic structure with the incommensurate propagation vector $\mathbf{q}_{\mathbf{m}}=(0.5+\tau, 0.5,0)$ in $2 \Pi / a$ units, with $\tau=0.0703 \pm 0.0005$ at $4.7 \mathrm{~K}$. This value is a little smaller but much more accurate than in the literature where it was obtained from a powder pattern [16]. The temperature variation of $\tau$ is given in figure $1:$ no clear locking of $\mathbf{q}_{\mathrm{m}}$ on simple commensurate values has been evidenced in the whole range of temperatures.

The temperature variation of the integrated intensity of the first-order satellite at $\mathbf{q}=(-0.5,-0.5+\tau$, 0 ) is consistent with the Néel temperature $T_{\mathrm{N}}=33 \mathrm{~K}$, see figure $1:$ at $11 \mathrm{~K}$, the neutron flux at its maximum reaches 1250 neutrons/s. A very small intensity is observed, at $\mathbf{q}=(1 / 2,1 / 2,0)$ less than $5 \%$ of $I\left(\mathbf{q}_{\mathrm{m}}\right)$ : it may corresponds to a $\lambda / 2$ contamination or to a residue of the commensurate $(1 / 2,1 / 2,0)$-type structure which coexists in an unannealed sample [16].

Below $27 \mathrm{~K}$, third-order satellites at $\mathbf{q}=(0.5 \pm 3 \tau$, $0.5,0)\left(\equiv 3 \mathbf{q}_{\mathrm{m}}+\mathbf{G}\right.$, where $\mathbf{G}$ is a reciprocal lattice vector) have been detected with an integrated intensity ratio of $\sim 5 \times 10^{-3}$ (at $4.7 \mathrm{~K}$ ) with regard to the first-order satellites (see Fig. 1). Their increasing inten-

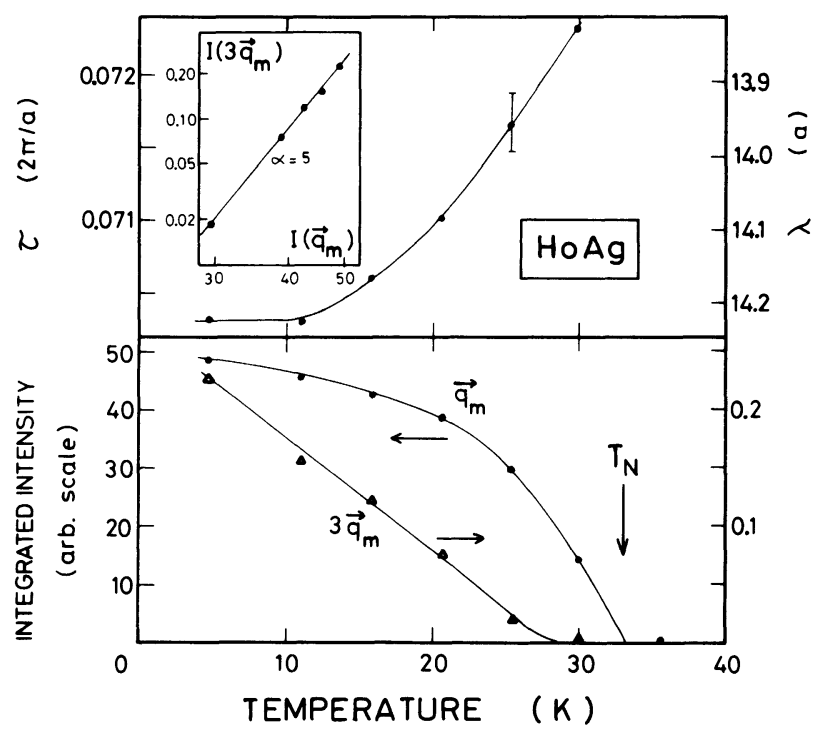

Fig. 1. - Upper part : temperature variation of $\tau$ in $\mathrm{HoAg}$ $(\lambda=1 / \tau$ is the associated wavelength); lower part : temperature variation of the intensity of elastic peaks at $\mathbf{q}_{\mathbf{m}}=$ $(-1 / 2,-1 / 2-\tau, 0)$ and $3 \mathbf{q}_{\mathrm{m}}=(-1 / 2,-1 / 2-3 \tau, 0)$ (in $2 \Pi / \mathrm{a}$ units); the inset shows the relative variation of $I\left(3 \mathbf{q}_{\mathrm{m}}\right)$ and $I\left(\mathbf{q}_{\mathrm{m}}\right)$; intensities are reduced to the same counting time. 
sity when the temperature is lowered corresponds to the progressive squaring-up of the modulated structure, which is quasi-sinusoidal between $27 \mathrm{~K}$ and $T_{\mathrm{N}^{*}}$ The relative temperature variation of $I\left(\mathbf{q}_{\mathrm{m}}\right)$ and $I\left(3 q_{m}\right)$ can be obtained by a log-log plot (see inset of Fig. 1) : a $I\left(3 \mathbf{q}_{\mathrm{m}}\right) \propto\left|I\left(\mathbf{q}_{\mathrm{m}}\right)\right|^{\alpha}$ law has been found, $\alpha$ being very close to 5 . This will be discussed in section 4 . No evidence for a saturation of the intensities has been observed at the lowest temperature : the structure is still not fully squared down to $4.7 \mathrm{~K}$.

A possible lattice modulation was also looked for by searching second-order satellites at $\mathbf{q}= \pm 2 \mathbf{q}_{\mathrm{m}}+$ $\mathbf{G}$, i.e. close to nuclear Bragg peaks. Effectively, elastic peaks have been observed at vectors such as $\mathbf{q}_{1}=$ $(-1,-1,-1+2 \tau), \mathbf{q}_{2}=(-1,-1,2 \tau), \mathbf{q}_{3}=$ $(-1 \pm 2 \tau,-1,0)$. Their normalized integrated intensity is noticeable, reaching $\sim 0.8$ for $\mathbf{q}_{2}$ at $11 \mathrm{~K}$, i.e. more than the third-order satellites intensity; it varies roughly as $\left|I\left(\mathbf{q}_{\mathrm{m}}\right)\right|^{\alpha}$ with $\alpha \approx 2.8$ up to $28 \mathrm{~K}$ when it becomes no measurable. However, the multiple scattering origin of these peaks could be unambigously proved by means of Renninger scans, i.e. by rotating the sample about the scattering vector : all second-order satellites present an oscillatory behaviour, with a minimum of the order of the background, which is not the case for first- or third-order satellites. Therefore, if a lattice modulation exists, it is smaller than the experimental sensitivity.

At last, possible coupling between equivalent incommensurate wave vectors $\mathbf{q}_{\mathbf{m}}$ have been also investigated by searching elastic peaks at vectors $\mathbf{q}=\mathbf{q}_{\mathbf{m}_{1}}+\mathbf{q}_{\mathbf{m}_{2}}$ for example. When present, such peaks have been always verified to be of multiple scattering origin.

\section{TmAg.}

3. 1 EXPERIMENTAL. - The neutron diffraction experiments were performed on the three-axis spectrometer IN2 of the I.L.L.; this spectrometer was chosen in order to reach temperatures as low as possible with regard to the Néel temperature of TmAg. The neutron wavelength was $2.36 \AA$. The TmAg single crystal was mounted in a variable temperature cryostat with the [001] axis vertical, $q$-scans were carried out between 1.6 and $16 \mathrm{~K}$ with a resolution better than 0.01 in reduced units in the investigated range. The lattice parameter was found to be $a=3.550 \AA$.

3.2 RESULTS. - As a first result, the structure previously obtained on a polycrystal [18] has been found again : between $1.6 \mathrm{~K}$ and the Néel temperature $T_{\mathrm{N}}=9.5 \mathrm{~K}, \mathrm{TmAg}$ has an amplitude modulated magnetic structure with the propagation vector $\mathbf{q}_{\mathbf{m}}=$ $(0.5+\tau, 0.5,0)$ with $\tau=0.0883 \pm 0.0005$ at $1.6 \mathrm{~K}$ in good agreement with reference [18] (the magnetic moments are parallel to the [001] direction). The temperature variation of $\tau$ is given on figure 2 : as for $\mathrm{HoAg}$, there is no clear evidence for a locking of the modulated structure on simple commensurate values, owing to the experimental accuracy.

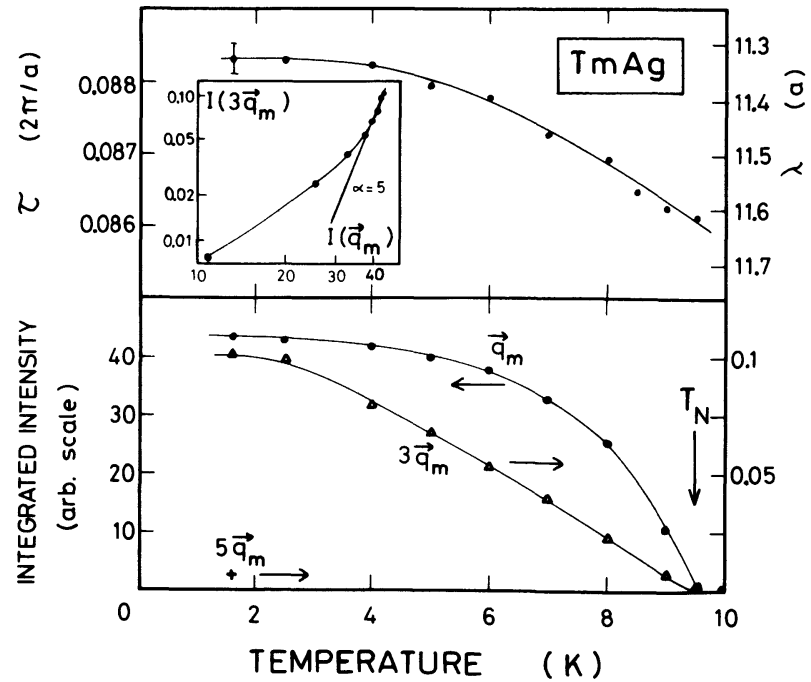

Fig. 2. - Upper part : temperature variation of $\tau$ in $\mathrm{TmAg}$ ( $\lambda=1 / \tau$ is the associated wavelength) ; lower part : temperature variation of the intensity of elastic peaks at $\mathbf{q}_{\mathrm{m}}=(1 / 2$, $1 / 2-\tau, 0), \quad 3 \mathbf{q}_{\mathrm{m}}=(1 / 2,1 / 2-3 \tau, 0)$ and $5 \mathbf{q}_{\mathrm{m}}=(1 / 2$, $1 / 2-5 \tau, 0$ ) (in $2 \Pi / \mathrm{a}$ units); the inset shows the relative variation of $I\left(3 \mathbf{q}_{\mathrm{m}}\right)$ and $I\left(\mathbf{q}_{\mathrm{m}}\right)$; intensities are reduced to the same counting time.

Figure 2 also shows the temperature of the integrated intensity of the first-order satellite $q=(0.5$, $0.5-\tau, 0)$ : at $1.6 \mathrm{~K}$, on its maximum, the neutron flux is about 4000 neutrons/s. As for HoAg, a small residue of the commensurate $(1 / 21 / 20)$-type antiferromagnetic structure subsists : the intensity of the $(0.5,0.5,0)$ line is less than $2 \%$ of $I\left(\mathbf{q}_{m}\right)$ (see Ref. [18]).

In figure 2 it is clear that third-order harmonics are sizeable immediately below $T_{\mathrm{N}}$, their integrated intensity reaching $\sim 2.3 \times 10^{-3}$ of $I\left(\mathbf{q}_{\mathrm{m}}\right)$ at $1.6 \mathrm{~K}$. Fifthorder satellites have been also detected at $1.6 \mathrm{~K}$, their intensity ratio being roughly $10^{-4}$ with regard to $\mathbf{q}_{m}$. Seventh-order harmonics have not been observed. For comparison, the intensity ratios were $I\left(3 q_{m}\right) / I\left(q_{m}\right) \sim$ $4 \times 10^{-3}$ and $I\left(5 \mathbf{q}_{\mathrm{m}}\right) / I\left(\mathbf{q}_{\mathrm{m}}\right)<10^{-4}$ for the isomorphous compound $\mathrm{TmCu}$ which has the same type of structure [20]. The relative variation of first- and third-order satellites seem to reach a $I\left(3 \mathbf{q}_{\mathrm{m}}\right) \propto$ $\left|I\left(\mathbf{q}_{\mathrm{m}}\right)\right|^{\alpha}$ law with $\alpha=5$ only below $6 \mathrm{~K}$ (see inset of Fig. 2), $\alpha$ being about 1.5 just below $T_{\mathrm{N}}$.

As for HoAg, second-order satellites at $2 \mathbf{q}_{m}$, i.e. close to nuclear Bragg peaks, have been also observed in $\mathrm{TmAg}$, with a normalized integrated intensity of $\sim 0.03$ at $1.6 \mathrm{~K}$ for $\mathbf{q}=(1,2 \tau, 0)$ for example; its intensity varies roughly as $\left|I\left(\mathbf{q}_{\mathrm{m}}\right)\right|^{\alpha}$ with $\alpha \sim 2.1$. However, such peaks have not been-detected systematically at all their theoretical position. Therefore, they are very probably due to multiple scattering processes as in HoAg. So it is for elastic peaks which have been observed at wave vectors such $\mathbf{q}=\mathbf{q}_{\mathrm{m}_{1}}+\mathbf{q}_{\mathrm{m}_{2}}$.

\section{Discussion.}

As a main result, the squaring-up of the modulated magnetic structure of $\mathrm{HoAg}$ and $\mathrm{TmAg}$ has been followed over a wide range of temperature (reduced 
temperature $t=T / T_{\mathrm{N}}$ varying between 0.15 and 1) by observing the increase of third-order satellites (and fifth-order ones for $\mathrm{TmAg}$ ) as the temperature decreases. However, the fully antiphase structure is not yet reached for $t=0.15$ : it would indeed lead to intensity ratios of $1 / 9$ and $1 / 25$ for $I\left(3 q_{m}\right)$ and $I\left(5 q_{m}\right)$ relatively to $I\left(q_{\mathrm{m}}\right)$. In $\mathrm{HoAg}$ and $\mathrm{TmAg}$, the obtained intensities have not been normalized to each other by taking into account the rare-earth form factor, the Lorentz factor, absorption, and extinction corrections, but only by reducing to the same counting time. Nevertheless the order of magnitude of the actual intensity ratios should be close to the values given above (sections 2 and 3 ) which are very far from the expected values for the antiphase structure.

This can be explained by calculating the magnetization $M$ as a function of the exchange field $H_{\text {ex }}$ taking into account the known crystalline electric field (CEF) scheme $[18,19]$. When decreasing the temperature below $T_{\mathrm{N}}$, the $M\left(H_{\mathrm{ex}}\right)$ curves are not linear but present a curvature which becomes larger and larger. The exchange field as well as the magnetization cannot remain sinusoidal, as if the curves were linear, and higher order harmonics have to grow. At $0 \mathrm{~K}$, due to the magnetic ground state, the $M\left(H_{\mathrm{ex}}\right)$ curve is a step function and $M$ cannot be smaller than the threshold value associated with the ground state : the magnetic structure then becomes a structure with constant moments. In HoAg and TmAg, calculations show that this situation is reached only for $t \lesssim 0.04$, explaining the absence of saturation of $I\left(3 \mathbf{q}_{\mathrm{m}}\right)$ at the lowest obtained temperatures $(t \sim 0.15)$.

On the other hand, the experimental value $\alpha=5$ in the $I\left(3 \mathbf{q}_{\mathrm{m}}\right) \propto\left|I\left(\mathbf{q}_{\mathrm{m}}\right)\right|^{\alpha}$ could be related to the relative temperature dependence of $m_{\mathbf{q}_{\mathrm{m}}}$ and $m_{3 \mathbf{q}_{\mathrm{m}}}$, the first Fourier components of the magnetization. This would require calculations which should include the CEF effects, a reasonable exchange coupling $J(q)$ and should be self-consistent with respect to the relation between $H_{\mathrm{ex}}$ and $M$ on each site [21] $(\alpha \neq 3$ generally except in the vicinity of the Néel temperature).

No locking of the incommensurate wave vector into a simple commensurate value has been evidenced in $\mathrm{HoAg}$ and $\mathrm{TmAg}$, contrarily to the case of thulium metal [10]. This may be related to the small $\tau$ value and the low rate of variation of $\tau$ with the temperature, which prevents $\tau$ to cross through simple commensurate values. On the other hand this locking could be favoured in the antiphase regime, because of the discontinuity of the moments in such a step function : this could be observed at reduced temperatures $t<0.04$. Anyway, more accurate experiments should be necessary to detect a possible locking.

The existence of a modulated lattice distortion has not been demonstrated in $\mathrm{HoAg}$ or $\mathrm{TmAg}$ : it remains below our experimental resolution. A possible explanation, valid at least for $\mathrm{HoAg}$, could be the weakness of the magnetoelastic coupling [22].

\section{References}

[1] Koehler, W. C., in Magnetic properties of rare earth metals, Elliott, R. J., ed. (Plenum Press, New York) 1972, p. 81-128.

[2] Rossat-Mignod, J., J. Physique Colloq. 40 (1979) C595.

[3] Shirane, G. and Takei, W. J., J. Phys. Soc. Japan, 17 Suppl. B III (1962) 35.

[4] Adam, A., Billerey, D., Terrier, C., Mainard, R., Regnault, L. P., Rossat-Mignod, J. and Meriel, P., Solid State Commun. 35 (1980) 1.

[5] Wada, N., Ubukoshi, K. and Hirakawa, K., J. Phys. Soc. Japan, 51 (1982) 2833.

[6] LiU, S. H., in Handbook on the Physics and chemistry of rare earths, Gschneidner, K. A. and Eyring, L., eds. (North-Holland Publishing Co, Amsterdam) 1978, Chap. 3, p. 233.

[7] Debray, D., Sougi, M. and Meriel, P., J. Chem. Phys. 56 (1972) 4325

[8] Атол, M., J. Chem. Phys. 48 (1968) 560.

[9] Morin, P. and SchmitT, D., J. Magn. Magn. Mat. 21 (1980) 243.

[10] Brun, T. O., Sinha, S. K., Wakabayashi, N., Lander, G. H., Edwards, L. R. and Spedding, F. H., Phys. Rev. B 1 (1970) 1251.

[11] Gignoux, D., Gomez Sal, J. C., Lemaire, R. and DE Combarieu, A., Solid State Commun. 21 (1977) 637.

[12] Barbara, B., Rossignol, M., Boucherle, J. X. and Vettier, C., Phys. Rev. Lett. 45 (1980) 938.
[13] Lassailly, Y., Vettier, C., Holtzberg, F., Flouquet, J., Zeyen, C. M. E. and Lapierre, F., Phys. Rev. B 28 (1983) 2880.

[14] Elliott, R. J., Phys. Rev. 124 (1961) 346.

[15] Koehler, W. C., Cable, J. W., Wollan, E. O. and Wilkinson, M. K., Phys. Rev. 126 (1962) 1672.

[16] Nereson, N., A.I.P. Conf. Proc. 10 (1973) 669.

[17] Nereson, N., J. Appl. Phys. 44 (1973) 4727.

[18] Morin, P. and Schmitt, D., J. Magn. Magn. Mat. 28 (1982) 188.

[19] Schmitt, D., Morin, P. and Pierre, J., Phys. Rev. B 15 (1977) 1698.

[20] Morin, P., Schmitt, D. and Vettier, C., J. Magn. Magn. Mat. 40 (1984) 287.

[21] It can be easily shown that the Fourier coefficients $H_{m q_{m}}$ of the exchange field are related to the Fourier coefficients $M_{n q_{m}}$ of the magnetization by the expression $H_{n \mathbf{q}_{\mathrm{m}}}=\frac{1}{g_{\mathrm{J}}^{2} \mu_{\mathrm{B}}^{2}} M_{n \mathbf{q}_{\mathrm{m}}} J\left(n \mathbf{q}_{\mathrm{m}}\right)$. By diagonalization of the Hamiltonian, the magnetization can then be calculated for each site, whence new Fourier coefficients $M_{n}$, and so on until reaching self-consistency. A similar procedure was used for thullium by FYnBo, P. B., J. Phys. F 7 (1977) 2179. This formalism is also well described by COQBLIN, B., in The electronic structure of rare earth metals and alloys (Academic Press, London) 1977, Chap. 3, p. 134.

[22] Morin, P. and Schmitt, D Phys. Rev. B 23 (1981) 2278. 